\title{
Blood derived treatment from two allogeneic sources for severe dry eye associated to keratopathy: a multicentre randomised cross over clinical trial
}

\author{
Emilio Campos, ${ }^{1}$ Piera Versura (D), ${ }^{1}$ Marina Buzzi, ${ }^{2}$ Luigi Fontana, ${ }^{3}$ \\ Giuseppe Giannaccare (D) ${ }^{1}$ Marco Pellegrini, ${ }^{1}$ Nico Lanconelli, 4 \\ Alessandra Brancaleoni, ${ }^{5}$ Fabiana Moscardelli, ${ }^{1}$ Stefano Sebastiani, ${ }^{1}$ Cristiana Vaselli, ${ }^{2}$ \\ Vanda Randi ${ }^{2}$
}

- Additional material is published online only. To view please visit the journal online (http://dx.doi.org/10.1136/ bjophthalmol-2019-314859).

'Ophthalmology Unit, DIMES, Alma Mater Studiorum University of Bologna and S. Orsola-Malpighi Teaching Hospital, Bologna, Italy ${ }^{2}$ Emilia Romagna Cord Blood Bank-Transfusion Service, AOU of Bologna Sant'OrsolaMalpighi Teaching Hospital, Bologna, Italy

${ }^{3}$ Ophthalmology Unit, Azienda USL-IRCCS di Reggio Emilia,

Reggio Emilia, Italy ${ }^{4}$ Department of Physics and Astronomy, University of Bologna, Bologna, Italy ${ }^{5}$ Ospedale degli Infermi di Rimini, Rimini, Italy

Correspondence to Dr Piera Versura, DIMESOphthalmology Unit, Univ Bologna, Bologna, Italy, Italy; piera.versura@unibo.it

Received 4 July 2019

Revised 24 September 2019 Accepted 30 October 2019
Check for updates

(c) Author(s) (or their employer(s)) 2019. No commercial re-use. See rights and permissions. Published by BMJ.

To cite: Campos E, Versura

$\mathrm{P}$, Buzzi $\mathrm{M}$, et al. $\mathrm{Br} J$

Ophthalmol

2020:104:1142-1147.

\section{ABSTRACT}

Aim To compare the efficacy of cord blood and peripheral adult donor blood serum eyedrops, controlled for growth factor content, in the treatment of severe dry eye diseases (DED) resistant to conventional therapy.

Methods This was a multicentre randomised, doublemasked, cross-over clinical trial. Sixty patients diagnosed as severe DED, associated to persistent corneal epithelial defects were randomised and equally assigned to group $A$ (treated with cord blood serum (CBS)) or group B (treated with PBS), eyedrops administered eight times/ day for 1 month. Primary outcome was the pretreatment and post-treatment change in corneal fluorescein staining. Secondary outcomes included the pretreatment and post-treatment change in Ocular Surface Disease Index (OSDI) questionnaire and Visual Analogue Score (VAS) of subjective symptoms, Schirmer I test, tear breakup time and conjunctival staining. Patients with relapse in signs or symptoms after further 2 months switched to the remaining group for one additional month. Data were statistically analysed $(p<0.05)$.

Results Corneal staining was more significantly reduced after the CBS treatment, both VAS and OSDI score reduction was observed in both groups, but group A reported significantly less grittiness and pain. Nineteen patients shifted in the crossover period, the within individual comparison confirmed a better recovery in the CBS treatment period. Reduction in epithelial damage was positively associated with epidermal growth factor, transforming growth factor $\boldsymbol{\alpha}$ and platelet-derived growth factor content. Levels of interleukins (IL-13) were positively associated with symptom decrease.

Conclusions Overall, DED signs improved after both CBS and PBS treatments, with potential advantages of CBS for subjective symptoms and corneal damage reduction.

Clinical trial registration NCT03064984.

\section{INTRODUCTION}

Corneal epithelium is essential in protecting the eye. Dry eye disease (DED) can result in mild to severe corneal epithelial defects, which can become persistent when a failure of the mechanisms promoting the normal turnover occurs, ${ }^{12}$ with associated compromised vision and discomfort or pain or even infection and cornea perforation.
The reason for the use of topically administered products prepared from the blood is mainly based on their content in substances mimicking the natural tears, which play a key role in maintaining the homeostasis of the ocular surface, such as growth factors (GF), cytokines, vitamins and nutrients. ${ }^{3} 4$

The most used products derive from peripheral blood taken from the patients themselves, and prepared in form of serum (autologous serum, AS) ${ }^{5}$ or platelet-rich plasma (Plasma Rich Platelet). ${ }^{6} 7$ Several published studies indicated that AS could be an effective treatment for DED, but this has been recently questioned ${ }^{5} 8$ as few randomised and controlled studies had been performed in support. Allogeneic bloodbased therapies from homologous sources have been more recently proposed for the treatment of epithelial defects with advantages as compared with autologous sources. ${ }^{7} 910$ Topical products prepared from adult donors' peripheral blood serum (PBS) as a source were tested in patients. ${ }^{11-13}$ Cord blood serum (CBS, collected at birth from placenta umbilical veins) based eye drops have also been proposed. ${ }^{14-18}$ Both sources were demonstrated to be efficient in the healing of epithelial defects, in the course of clinical trials having conventional therapies as control, and only one controlled study compared the effectiveness of autologous PBS versus allogeneic CBS. ${ }^{19}$ One major criticism may be that these studies had not characterised the content of each source for trophic substances, which was demonstrated to be different. ${ }^{20} 21$

The purpose of this study was to evaluate the effect of eye drops derived from two different allogeneic blood sources, namely CBS and PBS, characterised for their content in epitheliotropic GFs and cytokines. Patients suffering from DED associate to severe keratopathy and resistant to conventional therapy were randomised for CBS or PBS treatment, and results were correlated with the levels of GFs administered.

\section{METHODS}

\section{Study design}

This was a prospective, randomised, controlled, crossover, multicentre and interventional clinical study. 
The study was conducted according to the current legislation for clinical research and complied with the Health Insurance legislation for clinical trials in Italy. It was conducted between January 2017 and February 2018 in compliance with the International Conference on Harmonisation guidelines for Good Clinical Practice and the Declaration of Helsinki, and involved three centres in Italy (Ophthalmology Unit in Bologna, Reggio Emilia, and Rimini).

The study consisted of two periods. Period 1 was runned for 3 months, comprising 1 month of treatment and a 2-month followup after the end of treatment. Period 2 was runned for an additional months, and patients entered in this period as specified below.

Study visits were scheduled at day 1 (V1, visit 1/ randomisation), day 3-5 days (V2, visit 2/baseline, beginning of treatment), day $14 \pm 2(\mathrm{~V} 3$, visit 3/intermediate control), day 30 \pm 2 (V4, visit 4/end of the treatment, endpoint), day $90 \pm 2$ (V5, visit $5 /$ control after treatment suspension, end of period 1 ).

The patient eventually entering period 2 also was evaluated at day $93 \pm 3(\mathrm{~V} 6$, visit 6/baseline for period 2), day 107 $\pm 2(\mathrm{~V} 7$, visit 7/intermediate control), and finally at day $121 \pm 2$ (V8, visit8/end of the treatment of period 2, end of the study).

Patients were also contacted by telephone on day $8+2$ and if entered in period 2 on $115 \pm 2$ day, to ask about sensations at instillation.

\section{Treatment preparation}

The treatments under study comprised blood serum obtained from two different sources: CBS collected at birth and adult subject donor PBS. Serum from both sources were measured for levels of following GFs and interleukins (IL): IL-1 $\beta$, IL-4, IL-6, IL-10, IL-13, fibroblast GF (FGF), platelet-derived GF (PDGF), vascular endothelial growth factor (VEGF), nerve GF (NGF), epidermal GF (EGF), transforming GF (TGF)- $\alpha$, TGF- $\beta 1 / \beta 2 / \beta 3$, insulin GF (IGF)- 1 and IGF-2. Samples were evaluated using commercially available multiplex bead-based sandwich immunoassay kits (Bio-Rad Laboratories, California, USA), by means of the Bio-Plex Protein Array System (Bio-Rad Laboratories, California, USA) as previously described. ${ }^{21}$ The products were prepared, standardised, controlled and sealed in anonymous frozen unidose vials in the Transfusion Service, partner in the study, as it has been extensively described and reported previously. ${ }^{18} 21$ The explanation of the method is given in online supplementary file 1.

\section{Randomisation}

The assignment of the treatment in period 1 was performed through a computer generated randomisation schedule, stratified by study site, blind to the patient and the clinician, only known to the transfusion service personnel. The patient entered period 2 only in case of a corneal epithelial damage occurring at V5 and larger than that recorded at baseline, or subjective symptoms higher than that detected at baseline, and the treatment assigned belonged to the remaining arm. The treatment also in this period was only known to the transfusion service personnel, trained to keep data aside.

\section{Masking description}

The products under study were prepared, standardised, controlled and sealed in anonymous frozen vials in the Transfusion Service of the S. Orsola-Malpighi Hospital, our partner and collaborator in the study. The products had same physical and colour characteristics and could not be visually recognised. Boxes containing the vials reported a code of assignment only known by the transfusion service personnel.

\section{Inclusion criteria}

Eligible patients were $\geq 18$ years old and had moderate to severe symptomatic dry eye (defined according to the 2017 DEWS II classification ${ }^{22}$ ) with a baseline 12-item selfadministered Ocular Surface Disease Index (OSDI) score $\geq 23$ on a $0-100$ scale. Three subscales, interpreted as subscale A: ocular symptoms (three questions); subscale B: vision-related function (six questions); and subscale C: environmental triggers (three questions) were calculated and included in the statistic. Eligible patients had at least one eye with both of the following conditions at baseline: corneal damage coverage $>25 \%$ of total corneal area $(2 \mu \mathrm{L}$ instillation of $2 \%$ unpreserved sodium fluorescein and estimated as it will be described below), and a Schirmer score (without anaesthesia) $\geq 3$ and $\leq 10 \mathrm{~mm} / 5 \mathrm{~min}$ or three consecutive tear break-up time (TBUT) tests $\leq 10 \mathrm{~s}$. Subject must be on stable course of systemic therapy (oral immune suppressants consisting of steroids/cyclosporine) without plan to change or is not on systemic immunosuppressive therapy concomitant systemic treatments.

\section{Exclusion criteria}

Exclusion criteria were concurrent treatment with hypotensive drugs, ocular surgery in the 12 months preceding enrolment, history/active signs of ocular trauma, infection or inflammation (within 3 months of visit 1), active signs of ocular allergic disease or ocular herpes (within 2 years of visit 1); use of intraocular or periocular medications or punctum plugs (or lacrimal punctum cauterisation).

\section{Assessments}

During the screening visit, all study participants underwent a detailed ophthalmological examination including OSDI questionnaire and Visual Analogue Score (VAS), biomicroscopy, TBUT and corneal staining with fluorescein, conjunctival lissamine green staining (grade $0-18$ ), ${ }^{23}$ and Schirmer I test. All examination procedures and assessments were performed in the following order: patient assessment of symptoms, OSDI, TBUT, corneal staining, conjunctival staining, Schirmer test.

\section{Assessment of corneal damage}

The semiautomatic method for assessing corneal staining was developed by one co-author (NL) within the framework of the Image J software, provided in the public domain by the National Institutes of Health, Bethesda, Maryland, USA. ${ }^{24}$ The explanation of the method is given in online supplementary file 2 . The software has been implemented as a Macro for ImageJ and can be freely download from the website www.medphys.it.

\section{Treatment and assessments}

At randomisation patients were instructed to start administering 1-2 drops of sterile $0.9 \% \mathrm{NaCl}$ in each eye 2-6 times each day (as needed) for 3 days as a washout step. They were randomised (1:1) to CBS or PBS treatment, based on a computer generated randomisation scheme provided.

Treatments were dispensed by the Transfusion personnel, advising patients to store the vials in the $-20^{\circ} \mathrm{C}$ fridge, and thaw one vial at a time for the daily administration, which consisted of 8 drops/eye/daily. The content of GFs and ILs in the two treatments is summarised in table 1 . 
Table 1 The concentrations of growth factors (GF) and interleukins (ILs) in cord blood serum (CBS) and peripheral blood serum (PBS) treatments, administered to patients in this study. Values are expressed as $\mathrm{pg} / \mathrm{mL} /$ day, except for PDGF * $\mathrm{ng} / \mathrm{mL} /$ day. CBS treatment delivered higher content of both GFS and ILs as compared with PBS, with the exception of IGF1 and IGF2

\begin{tabular}{llllll}
\hline & \multicolumn{3}{l}{ CBS } & & \multicolumn{2}{l}{ PBS } \\
\cline { 2 - 3 } \cline { 5 - 5 } & Median & $95 \%$ Cl & Median & $95 \%$ CI \\
\hline EGF & 237.50 & 195.17 to 272.50 & 130.00 & 114.53 to 140.40 \\
\hline FGF & 122.00 & 117.69 to 133.00 & 25.30 & 10.76 to 42.57 \\
\hline IGF1 & 0.00 & 0.00 to 0.00 & 3.30 & 0.00 to 9.52 \\
\hline IGF2 & 4.25 & 3.56 to 5.44 & 23.95 & 19.36 to 29.08 \\
\hline NGF & 1.48 & 1.20 to 1.57 & 1.30 & 1.19 to 1.58 \\
\hline PDGF* & 2.04 & 1.79 to 2.36 & 1.29 & 1.19 to 1.37 \\
\hline IL-1- $\beta$ & 24.55 & 3.40 to 72.27 & 0.51 & 0.41 to 0.65 \\
\hline IL-4 & 0.27 & 0.18 to 0.37 & 0.18 & 0.090 to 0.27 \\
\hline IL-6 & 49.68 & 4.59 to 340.54 & 4.49 & 2.03 to 11.85 \\
\hline IL-10 & 2.40 & 2.27 to 2.73 & 1.96 & 1.80 to 2.52 \\
\hline IL-13 & 67.79 & 58.85 to 85.08 & 0.080 & 0.00 to 0.37 \\
\hline TGF- $\alpha$ & 14.22 & 11.57 to 15.96 & 4.04 & 3.10 to 4.86 \\
\hline VEGF & 81.50 & 33.14 to 134.58 & 36.60 & 31.20 to 42.64 \\
\hline
\end{tabular}

EGF, epidermal growth factor; FGF, fibroblast growth factor; IGF, insulin growth factor; NGF, nerve growth factor; PDGF, platelet-derived GF; TGF, transforming growth factor.

\section{Outcome measures and analyses}

The study eye was the eye with greater corneal epithelial damage at baseline, or the right eye if both eyes had equal damage. The primary endpoint was the change in corneal epithelium damage from V2 at V4 in the study eye. Secondary endpoints included change in OSDI, VAS, Schirmer test, TBUT, conjunctival staining, from V2 at V4. Satisfaction and tolerability to both treatments were recorded as described elsewhere ${ }^{25}$ at V4, and at V8 for subjects entered in period 2 of the study.

\section{Statistical analysis}

A sample size of 30 patients per treatment group (60 total) was determined according to a previous report ${ }^{18}$ adjusting the power value as $80 \%$ and type 1 error as 0.05 . The study was not powered for secondary endpoints.

The data were analysed using SPSS V.20.0. Prior to statistical analysis, data distribution was checked for normality. The Mann-Whitney U test was used to compare differences between two independent groups. The Wilcoxon signed-rank test was used when comparing paired measurements. The Spearman's correlation coefficient $\rho$ was calculated between primary and secondary outcome measures and the content of GFs and ILs in the treatment administered for each group. A p value of less than 0.05 was considered as statistically significant.

\section{RESULTS}

Forty-four women (median age 66 years, $42-82$ years $95 \% \mathrm{CI}$ ) and 16 men (median age 52.5 years, 39-82 years 95\% CI) were enrolled in the study and equally randomised in two groups. Demography of randomised patients is summarised in table 2 , and appeared homogeneous between groups (Levene's test $\mathrm{p}>0.05$ ).

Fifty-eight patients completed the period 1 study, while two were lost to follow-up before visit five due for reasons independent from the study.
Table 2 Demography of patients randomised in group A, assigned to CBS treatment, and group $B$, assigned to PBS treatment. Info on previous systemic or topical treatments is also given

\begin{tabular}{|lll}
\hline Group & A & B \\
\hline Treatment & CBS & PBS \\
\hline Females & 24 & 20 \\
\hline Age* & $65.5(61.5-69.0)$ & $66.5(61.3-70.0)$ \\
\hline Males & 7 & 9 \\
\hline Age* & $52.0(45.0-71.5)$ & $53.0(43.0-68.3)$ \\
\hline Rheumatoid arthritis (n) & 5 & 2 \\
\hline Sjogren's syndrome (n) & 9 & 7 \\
\hline GVHD (n) & 9 & 8 \\
\hline Systemic treatments (steroids) & 7 & 5 \\
\hline Systemic treatments (cyclosporine) & 3 & 4 \\
\hline Topical treatments & 30 & 30 \\
\hline Previous serum & 4 & 3 \\
\hline Steroids & 12 & 14 \\
\hline Cyclosporine & 2 & 2 \\
\hline Tear substitutes only & 20 & 18 \\
\hline Tear substitutes plus gel & 6 & 8 \\
\hline Tear substitutes plus ointment & 4 & 4 \\
\hline
\end{tabular}

*Years, median $(95 \% \mathrm{Cl})$.

CBS, cord blood serum; PBS, pheripheral blood serum.

After 1 month of treatment no change in the corneal staining was observed in four eyes (one from group A and three from group B), and a light worsening was observed in three eyes (one from group A and two from group B). A significant improvement in the corneal staining was observed in 53 of the 60 eyes (88.3\%), with the CBS treated group showing a statistically higher V4 versus V2 difference as compared with the PBS treated group $(p=0.02)$ (figure 1). Complete resolution of corneal damage was achieved in three of these 53 eyes $(5.5 \%$, two from group A and one from group B).

The subjective symptoms of discomfort significantly improved with respect to OSDI total score in both groups $(p<0.01)$. Only subset A score, regarding the subjective ocular symptoms, significantly lowered in the CBS treated group as compared with the PBS treated group $(p=0.03)$. No statistical significance was shown between groups as far as the vision-related function (subset $\mathrm{B}$ score) and environmental triggers (subset C score) symptoms.

Results for the VAS, Schirmer test, TBUT, and conjunctival staining are summarised in table 3. In particular, the Schirmer test and TBUT values did not change at V4 as compared with V2, with no statistically significant difference between group A and group B. The VAS and the conjunctival staining score were shown to decrease in both CBS and PBS treated patients at V4 as compared with V2 (p always $<0.01$ ) but without statistically significant difference between groups.

Between V4 and V5 patients returned to administer the same treatments used before enrolment, and in particular: 23 patients used only last generation tear substitutes; 15 patients hyaluronic acid-based tear substitutes associated to gels, and 22 tear substitutes hyaluronic acid-based tear substitutes associated to ointment.

A relapsing epithelial defect and/or a worsening in subjective symptoms occurred in 19 eyes $(31 \%, 8$ from the CBS treated group A and 11 from the PBS treated group B), after 2 months from the end of period 1. 


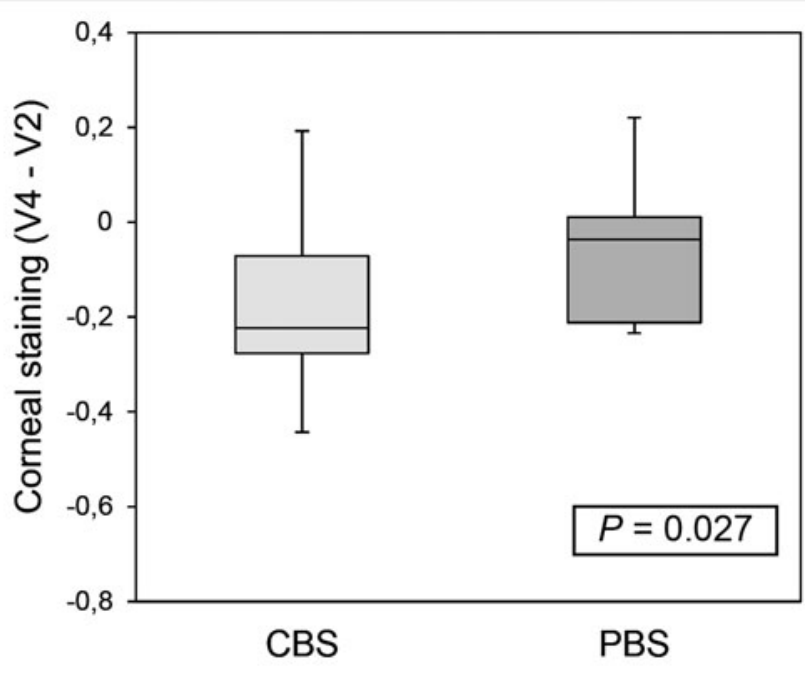

Figure 1 The change in corneal epithelium damage from V2 at V4 in the study eye is graphed. Reduction was observed in both groups, but the pre-difference and post-difference in the CBS treated group was more significant as compared with the PBS treated group ( $p=0.02)$. CBS, cord blood serum; PBS, peripheral blood serum.

A representative fluorescein stained slit-lamp image of a patient running both period 1 and period 2 of the study is given in figure 2. Data were analysed in a within-individual comparison between changes observed in the two periods of treatment, and results are summarised in figure 3. In particular, the CBS treatment resulted in a significantly higher reduction of

Table 3 OSDI with the three subset score (sub A, B, C), VAS score, Schirmer test, tear break up time (TBUT) and conjunctival staining score before (V2) and after (V4) 1-month CBS and PBS treatment. No change was observed for the Schirmer test and TBUTvalues, whereas a reduction was shown for OSDI, conjunctival staining and VAS, but without a statistically significant difference between treatments. Among OSDI subset scores, only sub A significantly lowered in the CBS treated group as compared with the PBS treated group $(p=0.03)$. Values expressed as median $(95 \% \mathrm{Cl})$

\begin{tabular}{|c|c|c|c|}
\hline \multicolumn{2}{|l|}{ CBS } & \multicolumn{2}{|l|}{ PBS } \\
\hline V2 & V4 & V2 & V4 \\
\hline \multicolumn{2}{|l|}{ OSDI score } & \multicolumn{2}{|l|}{ OSDI score } \\
\hline $52(47-60)$ & $31(28-37)$ & $48(38-59)$ & $38(29-44)$ \\
\hline SUB A 24 (13-30) & $15(10-16)$ & $28(20-32)$ & $21(14-34)$ \\
\hline SUB B 13 (8-19) & $8(4-10)$ & $12(8-14)$ & $8(4-14)$ \\
\hline SUB C $14(10-20)$ & $11(6-18)$ & $12(8-20)$ & $8(6-20)$ \\
\hline \multicolumn{2}{|l|}{ VAS score } & \multicolumn{2}{|l|}{ VAS score } \\
\hline \multicolumn{2}{|l|}{$7.4(6.5-7.8)$} & \multicolumn{2}{|l|}{$5.0(4.5-5.8)$} \\
\hline \multicolumn{2}{|l|}{ Schirmer test } & \multicolumn{2}{|l|}{ Schirmer test } \\
\hline $5.0(4.1-7.0)$ & $5.0(3.1-6.8)$ & $4.0(2.7-6.2)$ & $5.0(4.0-6.0)$ \\
\hline \multicolumn{2}{|l|}{ TBUT } & \multicolumn{2}{|l|}{ TBUT } \\
\hline $3.0(2.0-4.0)$ & $3.0(2.0-4.2)$ & $3.0(1.0-4.0)$ & $2.5(2.0-4.0)$ \\
\hline \multicolumn{2}{|c|}{ Conjunctival staining score } & \multicolumn{2}{|c|}{ Conjunctival staining score } \\
\hline $9.0(6.0-11.0)$ & $6.0(3.0-7.4)$ & $11.0(7.0-12.0)$ & $7.0(5.0-9.0)$ \\
\hline
\end{tabular}

CBS, cord blood serum; OSDI, Ocular Surface Disease Index; PBS, peripheral blood serum; TBUT, Tear Break Up Time; VAS, Visual Analogue Score.

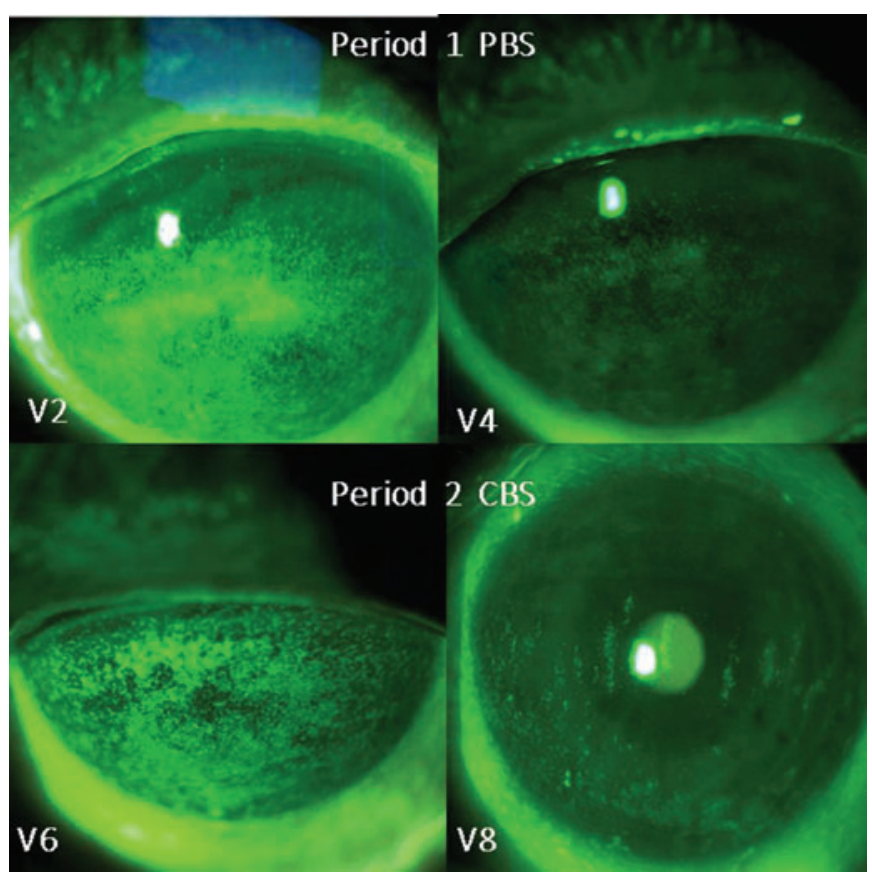

Figure 2 Slit-lamp photographs with fluorescein staining in patient running both period 1 and period 2 of the study. Before PBS treatment, the corneal epithelium was stained diffusely. (Top left). One month after treatment, severity of the damage decreased especially in the central area (top right). Two months after the end of the treatment, the level of epithelial damage worsened and patient entered period 2 (bottom left). After 1 month of CBS treatment, a diffuse decrease in staining was observed in all areas. In this patient, a better improvement was observed after the CBS treatment as compared with the PBS one. CBS, cord blood serum; PBS, peripheral blood serum.

corneal damage, either as administered in period 1 or period 2 (figure 2, upper part). The CBS treatment resulted in a significant higher reduction of both OSDI and VAS score, only when administered in period 1 (figure 2 middle and bottom, respectively).

The conjunctival staining was reduced in both period 1 and period 2 but without differences between periods. TBUT and Schirmer test values did not change in this period of the study.

A direct relationship was found between the corneal damage reduction and the content of EGF, TGF $\alpha$, PDGF, FGF, and IL13 (Spearman's $\rho$ respectively $-0.583,-0.509,-0.512,-0.467$ and $-0.427 ; p<0.01)$, whereas an indirect relationship was shown for the content of IGF1 and IGF2 ( $\rho 0.550$ and 0.491 , respectively). IL13 was found in direct relationship with the OSDI subset A and B reduction $(\rho-0.440$ and -0.476 , respectively, $p<0.01)$.

No adverse event was recorded in the study. The overall satisfaction and tolerability for both treatments was very high, and only three patients (one from group A and two from group B in period 1) reporting occasional symptoms as mild blurring at instillation, which disappeared after less than $3 \mathrm{~min}$ and which did not prevent them from continuing and completing the study successfully.

\section{DISCUSSION}

This is the first double blind, randomised, cross over, and controlled clinical trial, comparing PBS from healthy donors vs CBS eye drops for the treatment of patients affected by DED with associated severe corneal damage, reporting whether clinical outcomes may be correlated with the content of epitheliotropic substances in the treatments. 

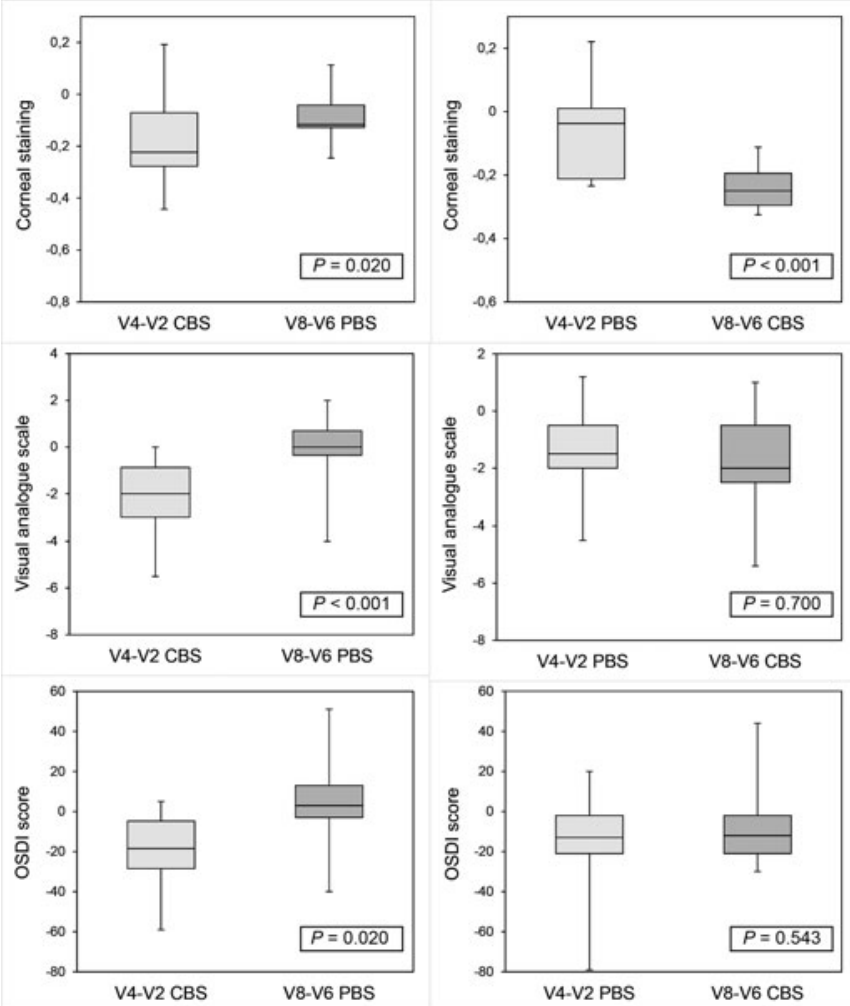

Figure 3 Within individual comparison in the cross over period of the study. Changes in period 1 (differences between V4 and V2) were compared with changes in period 2 (differences between V8 and V6) with respect of corneal damage (top), Visual Analogue Score (VAS) scale (middle) and Ocular Surface Disease Index (OSDI) score (bottom) in the same group of individuals entering the period 2 of the study. In the left column results for the eight patients receiving CBS in period 1 and $\mathrm{PBS}$ in period 2. In the right column results for the 11 patients receiving PBS in period 1 and CBS in period 2. The CBS treatment resulted in a higher reduction of corneal damage, either as administered in period 1 (top left) or period 2 (top right). The CBS treatment resulted in a higher reduction of VAS score only when administered in period 1 (middle left) but not when administered in period 2 (middle right). The same trend was observed for the OSDI score (bottom left and right). CBS, cord blood serum; PBS, peripheral blood serum.

Data from this study confirmed that both PBS and CBS eye drops are safe and effective in reducing the subjective symptoms of discomfort and the corneal damage after 1 month of treatment, as suggested by previous works that evaluated these treatments separately. The CBS treatment resulted in a higher reduction of corneal damage as compared with PBS in any period administered, which was confirmed also in the within-individual comparison. A relapsing epithelial damage and/or symptom degree occurred in one third of treated patients, feasibly related to the chronic nature of the disease, and perhaps to the relatively short period of treatment with the products in study.

Autologous blood derived eye drops are still widely used but not all patients are good candidates as donors due to underlying systemic inflammatory diseases, age, and other types of comorbidities. ${ }^{26}$ Allogenic blood sources for the preparation of topical treatments are conceptually preferable as compared with AS due to several reasons. 9101227 They avoid invasiveness to the patient, who could in time not like the repeated withdrawals. They can be prepared, controlled, validated for safety profile and standardised in advance, then stored frozen until the dispensation, with an unlimited availability in principle. Despite the current legislation for the ABO matching is applied worldwide also to blood derived eye drops, inherent risks present in homologous transfusions persist despite all efforts to avoid them. To the best of our knowledge, no adverse event was reported so far in the literature.

Allogenic serum from patients' relatives was used in a previous work to treat persistent epithelial defects, which healed within 2 weeks in about the half. ${ }^{11}$ Eye drops from healthy male donors matched for the $\mathrm{ABO}$ were administered for two to 4 weeks, and a partial or full epithelial healing, along with subjective symptom improvement was observed in patients with DED with only mild but not persistent defects. ${ }^{12}$ Another study reported the efficacy of a 4 weeks treatment of patients with severe dry eye associated to ocular graft versus host disease (GVHD) with healthy donor PBS. ${ }^{13}$

The efficacy of CBS treatment was demonstrated in patients suffering from DED,${ }^{14}$ neurotrophic keratitis ${ }^{15}$ recurrent corneal erosions, ${ }^{16}$ persistent epithelial defects, ${ }^{17} 1828$ in comparison or not with tear substitutes. Only one controlled and randomised clinical trial demonstrated CBS drops to be more effective in decreasing symptoms and corneal damage as compared with AS eye drops in Sjogren's syndrome patients. ${ }^{19}$

Despite the many clinical studies utilising serum derived eyedrops, there is no internationally harmonised method for preparing the products. ${ }^{29} 30$ Results from the present study were obtained with products standardised for the procedure of preparation ${ }^{18}$ and analysed for their content in selected trophic factors, ${ }^{21}$ which are present in both products, but at a higher levels in CBS.

Several GFs are present in normal human tears. ${ }^{31}$ For instance, the levels of EGF has been estimated in the range $3-8 \mathrm{ng} / \mathrm{mL}$ in unstimulated tear samples, with a threefold increase directly after wounding, subsequently returning to basal levels as shown in in vivo experiments. ${ }^{32}$

A significantly increased proliferation of corneal epithelial cells is produced in vitro at concentrations in the range $0.1-5 \mathrm{ng} / \mathrm{mL}^{33}$ and topical application of $500 \mu \mathrm{g} / \mathrm{mL}$ EGF can improve the rate of healing of corneal wounds in animal models. ${ }^{34}$

Interestingly, the amount of GFs in both CBS and PBS treatments, successful in our study, were in the range of $\mathrm{pg} / \mathrm{mL} /$ day. Beyond the obvious hardly comparable settings, this difference may be due to intrinsic properties of engineered GFs, ${ }^{35}$ supporting the higher efficacy of GFs from a natural source. For CBS in particular, the combination of GFs is the outcome of a period of high metabolic demand, and it might therefore represent the most powerful combination of trophic factors. The role and activity of each GF and IL depends on the microenvironment and the system, and there may be many other proinflammatory or anti-inflammatory agents in both sources that were not measured in our study. To discuss the role of each single GF and IL is out of the purpose of this work (for references $\operatorname{see}^{2}{ }^{21}$ ), but it's to highlight here the role of IL-13, a natural anti-inflammatory cytokine shown in this study to be correlated with improvement of subjective symptoms. Under these premises, the hypothesis is that this natural combination of epitheliotropic factors might increase the efficacy of the treatment, although this issue strongly deserves and still needs further studies.

In conclusion, data from this study support the clinical use of allogeneic PBS eye drops from adult donors, as a new and safe blood product effective in decreasing epithelial corneal damage already after 4 weeks of treatment. CBS eye drops seem to be more effective not only in reducing corneal epithelial damage but also in decreasing symptoms. Due to CBS sampling at delivery, its availability may be more restricted as compared with PBS. For this reason, a hypothesis could be to preferably dispense CBS in those cases where severe and unsustainable symptomatology is associated. 
Acknowledgements The authors thank Simonetta Nucci Director of Transfusion Service - Ospedale degli Infermi, Rimini and Roberto Baricchi, Director of Transfusion Service-Azienda USL - IRCCS di Reggio Emilia for eye drop management. Authors also thank Elisa Bergantin and Silvia Santandrea, Emilia Romagna Cord Blood Bank - S.OrsolaMalpighi Teaching Hospital for their excellent technical support to the eye drop preparation.

Contributors EC, PV, LF: design of the work. MB, CV, LF, GG, MP, AB, SS, FM: data collection. PV, LF, MP, GG, NL, SS: data analysis and interpretation. PV, MB, MP, NL, VR: drafting the article. EC, PV, LF, GG: critical revision of the article. All authors: final approval of the version to be published.

Funding This work was supported by Emilia Romagna Regional Blood Center (grant protocol n. 60400 - 2015 to Marina Buzzi).

Competing interests None declared.

Patient consent for publication Obtained.

Ethics approval This study protocol was approved by the Comitato Etico Indipendente Azienda Ospedaliero Universitaria di Bologna, Policlinico S. OrsolaMalpighi (local Ethical Committee), and by the Ethical Committees of the two other clinical sites involved (Reggio Emilia and Rimini).

Provenance and peer review Not commissioned; externally peer reviewed.

Data availability statement All data relevant to the study are included in the article or uploaded as supplementary information.

ORCID iDs

Piera Versura http://orcid.org/0000-0002-2951-5337

Giuseppe Giannaccare http://orcid.org/0000-0003-2617-0289

\section{REFERENCES}

1 Liu C-Y, Kao WW-Y. Corneal epithelial wound healing. Prog Mol Biol Trans/ SCi 2015;134:61-71.

2 Ljubimov AV, Saghizadeh M. Progress in corneal wound healing. Prog Retin Eye Res 2015:49:17-45.

3 Giannaccare $G$, Versura P, Buzzi M, et al. Blood derived eye drops for the treatment of cornea and ocular surface diseases. Transfus Apher Sci 2017;56:595-604.

4 Geremicca W, Fonte C, Vecchio S. Blood components for topical use in tissue regeneration: evaluation of corneal lesions treated with platelet lysate and considerations on repair mechanisms. Blood Transfus 2010;8:107-12.

5 Pan Q, Angelina A, Marrone M, et al. Autologous serum eye drops for dry eye. Cochrane Database Syst Rev 2017;2.

6 Alio JL, Rodriguez AE, Ferreira-Oliveira R, et al. Treatment of dry eye disease with autologous platelet-rich plasma: a prospective, interventional, Non-Randomized study. Ophthalmol Ther 2017:6:285-93.

7 Anitua E, de la Fuente M, Muruzabal F, et al. Plasma rich in growth factors (PRGF) eye drops stimulates scarless regeneration compared to autologous serum in the ocular surface stromal fibroblasts. Exp Eye Res 2015;135:118-26.

8 Pan Q, Angelina A, Zambrano A, et al. Autologous serum eye drops for dry eye. Cochrane Database Syst Rev 2013:CD009327.

9 Badami KG, McKellar M. Allogeneic serum eye drops: time these became the norm? $\mathrm{Br}$ J Ophthalmol 2012:96:1151-2.

10 van der Meer PF, Seghatchian J, de Korte D. Autologous and allogeneic serum eye drops. The Dutch perspective. Transfus Apher Sci 2015;53:99-100.

11 Chiang C-C, Chen W-L, Lin J-M, et al. Allogeneic serum eye drops for the treatment of persistent corneal epithelial defect. Eye 2009;23:290-3.
12 Harritshøj LH, Nielsen C, Ullum H, et al. Ready-made allogeneic ABO-specific serum eye drops: production from regular male blood donors, clinical routine, safety and efficacy. Acta Ophthalmol 2014;92:783-6.

$13 \mathrm{Na}$ K-S, Kim MS. Allogeneic serum eye drops for the treatment of dry eye patients with chronic graft-versus-host disease. J Ocul Pharmacol Ther 2012;28:479-83.

14 Yoon K-C, Im S-K, Park Y-G, et al. Application of umbilical cord serum eyedrops for the treatment of dry eye syndrome. Cornea 2006;25:268-72.

15 Yoon K-C, You I-C, Im S-K, et al. Application of umbilical cord serum eyedrops for the treatment of neurotrophic keratitis. Ophthalmology 2007;114:1637-42.

16 Yoon K-C, Choi W, You I-C, et al. Application of umbilical cord serum eyedrops for recurrent corneal erosions. Cornea 2011:30:744-8.

17 Vajpayee RB, Mukerji N, Tandon R, et al. Evaluation of umbilical cord serum therapy for persistent corneal epithelial defects. Br J Ophthalmo/ 2003:87:1312-6.

18 Versura P, Profazio V, Buzzi M, et al. Efficacy of standardized and quality-controlled cord blood serum eye drop therapy in the healing of severe corneal epithelial damage in dry eye. Cornea 2013;32:412-8.

19 Yoon K-C, Heo H, Im S-K, et al. Comparison of autologous serum and umbilical cord serum eye drops for dry eye syndrome. Am J Ophthalmol 2007;144:86-92.

20 Versura P, Buzzi M, Giannaccare G, et al. Targeting growth factor supply in keratopathy treatment: comparison between maternal peripheral blood and cord blood as sources for the preparation of topical eye drops. Blood Transfus 2016;14:145-51.

21 Buzzi M, Versura P, Grigolo B, et al. Comparison of growth factor and interleukin content of adult peripheral blood and cord blood serum eye drops for cornea and ocular surface diseases. Transfus Apher Sci 2018:57:549-55.

22 Craig JP, Nichols KK, Akpek EK, et al. TFOS DEWS II definition and classification report. Ocul Surf 2017:15:276-83.

$23 \mathrm{NEI} /$ Industry Grading System. American Academy of ophthalmology, 2014. Available: https://www.aao.org/image/neiindustry-grading-system [Accessed 15 Mar 2019].

24 pubmeddev. NIH Image to ImageJ: 25 years of image analysis. [Nat Methods, 2012. Available: https://www.ncbi.nlm.nih.gov/pubmed [Accessed 15 Mar 2019].

25 Fariselli C, Giannaccare G, Fresina M, et al. Trehalose/hyaluronate eyedrop effects on ocular surface inflammatory markers and mucin expression in dry eye patients. Clin Ophthalmol 2018:12:1293-300.

26 Valentini CG, Nuzzolo ER, Orlando N, et al. Cytokine profile of autologous platelet-derived eye drops in patients with ocular chronic graft-versus-host disease. Vox Sang 2016;110:189-92.

27 Anitua E, de la Fuente M, Merayo-Lloves J, et al. Allogeneic blood-based therapies: hype or hope?Eye 2017;31:509-10.

28 Yoon K-C, Heo H, Jeong I-Y, et al. Therapeutic effect of umbilical cord serum eyedrops for persistent corneal epithelial defect. Korean J Ophthalmol 2005;19:174-8.

29 Marks DC, van der Meer PF. Biomedical excellence for safer transfusion (best) collaborative. serum eye drops: a survey of international production methods. Vox Sang 2017;112:310-7

30 van der Meer PF, Seghatchian J, Marks DC. Quality Standards, safety and efficacy of blood-derived serum eye drops: a review. Transfus Apher Sci 2016;54:164-7.

31 Klenkler B, Sheardown H, Jones L. Growth factors in the tear film: role in tissue maintenance, wound healing, and ocular pathology. Ocul Surf 2007;5:228-39.

32 Sheardown $\mathrm{H}$, Cheng YL. Tear EGF concentration following corneal epithelial wound creation. J Ocul Pharmacol Ther 1996;12:239-43.

33 Imanishi J, Kamiyama K, Iguchi I, et al. Growth factors: importance in wound healing and maintenance of transparency of the cornea. Prog Retin Eye Res 2000;19:113-29.

34 Sheardown $\mathrm{H}$, Wedge $\mathrm{C}$, Chou $\mathrm{L}$, et al. Continuous epidermal growth factor delivery in corneal epithelial wound healing. Invest Ophthalmol Vis Sci 1993:34:3593-600.

35 Mitchell AC, Briquez PS, Hubbell JA, et al. Engineering growth factors for regenerative medicine applications. Acta Biomater 2016:30:1-12. 